\title{
NONLINEAR FIR MAGNETO-PHOTOCONDUCTIVITY ON QUASI-BOUND COULOMB STATES OF LIGHT HOLES IN HIGH PURITY p-Ge
}

\author{
G. Jungwirt, R. Kropf, and W. Prettl \\ Institut für Angewandte Physik \\ Universität Regensburg \\ 8400 Regensburg, Germany
}

Received April 11, 1991

\begin{abstract}
The far-infrared magneto-photoconductivity due to optical transitions from the acceptor ground state into quasi-bound Coulomb states in $\mathrm{p}$-Ge has been investigated at low temperatures as a function of intensity applying a high-power $\mathrm{cw}$ molecular laser. For intensities above about $1 \mathrm{~mW} / \mathrm{cm}^{2}$ the photoconductive signal shows a square root dependence on intensity, which is attributed to nonlinear free carrier capture in the low compensated material. The experimental results are analyzed in terms of a rate equation model yielding the kinetic parameters of the carrier generation- and recombination process involved.
\end{abstract}

Key words : semiconductors, shallow acceptors, far-infrared magnetospectroscopy, optical saturation

\section{Introduction}

The kinetics of charge carriers bound to shallow impurities in high-purity semiconductors may be studied by saturation spectroscopy in the far-infrared (FIR) spectral range. Very detailed measurements have been performed on shallow donor transitions of $\mathrm{n}$-GaAs epitaxial layers in an external magnetic field at low temperatures. As a function of the laser intensity incident on the sample, the absorption coefficient $[1,2]$ and the photoconductive signal due to optical transitions to excited impurity states and cyclotron resonance $[3,4,5]$ have been measured for various magnetic field strengths. The experimental results were analyzed in terms of appropriate rate equation models based on the photothermal free carrier generation process [6]. At temperatures below liquid helium temperature where the thermal ionization rate of excited impurity states is strongly reduced, excited state impact ionization 
has been proved to be the dominant mechanism leading to a photoconductive signal [7]. High purity n-GaAs epitaxial layers are typically 50 to 90 pc compensated. Therefore a substantial optically generated population in excited donor states can be accumulated at free electron concentrations less than the density of compensating acceptors. In this case the recombination rate is a linear function of the density of the free carriers and both the absorption and the resonant photoconductive signal show, as long as impact ionization may be neglected, a simple saturation behaviour. governed by the usual saturation intensity.

In the present study the magneto-photoconductivity spectra of high purity and low compensated p-Ge are surveyed and the intensity dependence of photoionization across resonant transitions to quasibound Coulomb states of shallow acceptors is investigated using a high power molecular laser. The photoconductive signals show a nonlinear dependence on intensity which cannot be described by a simple saturation behaviour. As the compensation ratio of the present samples is as small as $1 \mathrm{pc}$, the optically generated free hole concentration $p$ may exceed the compensating shallow donor density $N_{D}$ even for very low intensities. The recombination rate then becomes proportional to the square of free carrier concentration, causing a square root dependence of the photoconductive signal on intensity. In addition the resonant signal depends nonlinearly on the applied electric bias voltage and the linewidth of resonant transitions narrows upon increasing voltage. This indicates the effect of excited state impact ionization, which depopulates the optically excited quasi-bound states and contributes to the increase of free carriers in the valence band.

\section{Experimental Technique}

The measurements were performed on a high-purity p-Germanium sample containing predominantly the shallow acceptor boron and to a smaller amount aluminium with concentration ratio $n_{A l} / n_{B}=0.1$. Therefore all spectral structures of appreciable strength were due to boron. The effective acceptor concentration and the compensation ratio were $N_{A}-N_{D}=2.5 \cdot 10^{12} \mathrm{~cm}^{-3}$ and $N_{D} / N_{A}=0.01$, respectively. Ohmic contacts were made by alloying $\mathrm{Al}$ on opposite edges. The sample was mounted in an immersion cryostate in the center of a superconducting magnet. The measurements were carried out in Faraday configuration with the magnetic field perpendicular to the electric bias field and normal to the [111] face of the crystal. An electrically pulsed low pressure $\mathrm{CO}_{2}$-laser was used to pump a FIR-molecular laser at different wavelengths between $\lambda=57 \mu \mathrm{m}$ and $96 \mu \mathrm{m}$. The pulse duration was $150 \mu \mathrm{s}$, which is longer than any expected relaxation time ensuring steady-state condition during 
irradiation. The intensity of the laser beam in the sample was determined by a calibrated pyroelectric detector taking into account the losses in the windows and the reflection at the crystal surface. In any case the absorption coefficient times the sample thickness was much smaller than one, thus variations of the intensity in the sample may be neglected. The photoconductive signal was measured with a standard load resistor circuit and recorded by boxcar technique. The load resistor was chosen to be much smaller than the sample resistance, thus the relative change of the voltage across the sample, $\Delta V / V$, is proportional to the change of the sample conductance $\Delta \sigma$. Assuming that the hole mobility is not much changed, it follows that the voltage change is proportional to the optically generated free carrier concentration $\Delta p$. The bias voltage was kept well below the impact ionization threshold of shallow acceptors to avoid impact ionization nonlinearities [8].

\section{Excited State Spectra}

The photoconductive spectra for several laser lines up to a magnetic field strength of $6.5 \mathrm{~T}$ are shown in Fig.1. The spectra show pairs of strong lines and additional weaker structures, emerging out of a continuous background signal which decreases with increasing magnetic field strength. The lines are due to transitions from the acceptor ground state to quasi-bound Coulomb states $[9,10]$, being formed in the presence of a magnetic field below each Landau level. In the spherical approximation the Landau levels may be characterized by $N_{n_{a}}$, where $N=n_{a}+M_{j}+3 / 2$ combines the oscillator quantum number $n_{a}$ with the Bloch state angular momentum $M_{j}[11]$.

The orbital angular momentum quantum number $m_{l}$ is given by $m_{l}=n_{a}-n_{b}$, where $n_{b}$ is the second oscillator quantum number; the total angular momentum is $m=m_{l}+M_{j}$. Landau levels with different $m$ but having the same $N_{n_{a}}$ are degenerate due to the translational invariance of the cyclotron orbit perpendicular to the magnetic field. This degeneracy is lifted in the presence of a Coulomb attraction center leading to series of bound and quasi-bound states of different energy for different $m$, which may be counted by the number of nodes $\kappa$ of the wavefunction along the magnetic field [12]. On the other hand excited acceptor states with total angular momentum $m<N-3 / 2$ are degenerated with the continuum of lower Landau levels with the same angular momentum to which they are coupled by the Coulomb potential, thus autoionization of shallow impurities excited in such states may occur and, therefore, these states are called quasi-bound [9] or metastable [12].

The pairs of strong lines in Fig. 1 belong to the light hole Landau levels with $M_{j}= \pm 3 / 2$. In agreement to previous measurements 


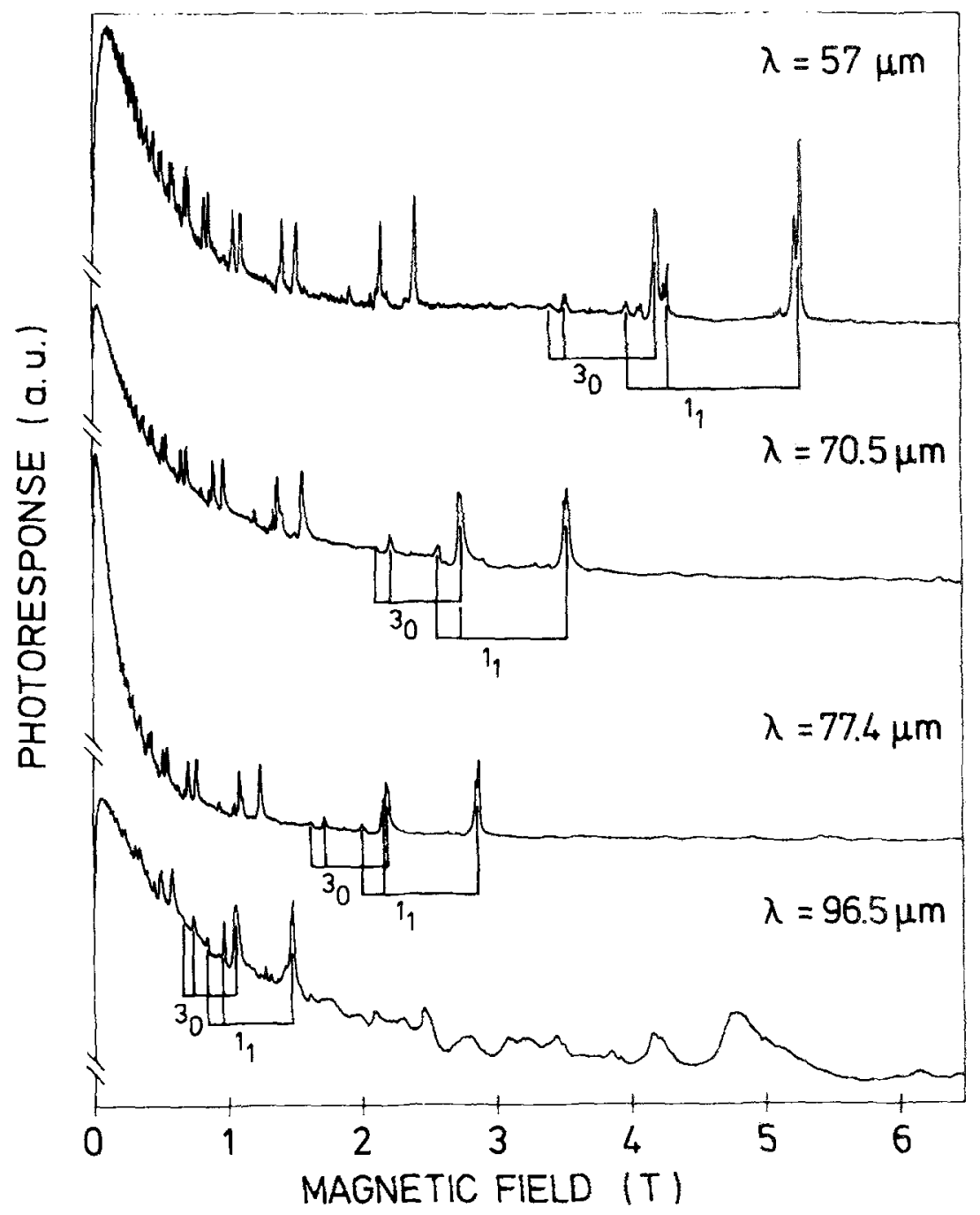

Fig.1 - Photoconductivity spectra of p-Ge for different FIR-laser lines ( wavelength $\lambda=57 \mu \mathrm{m}, \lambda=70.5 \mu \mathrm{m}, \lambda=77.4 \mu \mathrm{m}$, and $\lambda=96.5 \mu \mathrm{m}$ ) as a function of magnetic field for $4.2 \mathrm{~K}$. The sharp lines are attributed to transitions to quasi-bound Coulomb states. The sets of states belonging to the light hole Landau level $1_{1}$ and $3_{0}$ are marked.

at constant magnetic field using a Fourier-spectrometer, we observe series of lines belonging to common light hole Landau levels $N_{n_{a}}[10]$. 
Up to three lines of each series could uniquely be identified. For the $1_{1}$ and $3_{0}$-Landau levels the series are indicated by bars with a common base line in Fig.1.

The peak positions of all observed line series are plotted in Fig.2 together with the results of Fourier spectroscopy measurements as a function of the magnetic field strength. For the sake of clearity energy levels with $M_{j}=3 / 2$ and $M_{j}=-3 / 2$ are presented seperately. Calculated Landau levels relative to the zero magnetic field ground state binding energy of boron are shown by the broken lines [13]. The resonances detected by Fourier spectroscopy and by laser magneto-spectroscopy are shown as points and crosses, respectively, being in very good agreement. With the laser magnetomeasurements resonances up to a Landau quantum number of $N=16$ can be resolved. As the increase of binding energy of the acceptor ground state with rising magnetic field strength is not known, the correct energy separation between the Landau levels and the $1 S_{3 / 2}$ ground state cannot be plotted, thus the continuum threshold of each Coulomb resonance series must be somewhat above the broken lines in Fig.2.

\section{Nonlinear Photoconductivity}

The observed nonlinearities of the photoconductive signal will be discussed for the optical transition from the ground state to the lowest quasi-bound state belonging to the $1_{1}$-Landau level. The relative photoconductive signal $\Delta V / V$ measured with a bias voltage of $1.5 \mathrm{~V}$ is displayed in Fig. 3 as a function of magnetic field strength for various intensities.

The line shows a fine structure which is observed for final states with not too large Landau quantum numbers ( see Fig.1). The fine structure is due to close lying final states with the same $\kappa$ but different total angular momentum quantum number $m$ which, as noted above, have different energies. The resonance shown in Fig. 3 contains at least two transitions.

The resonant peak signals for various intensities have been evaluated by subtracting the continuous background photoconductivity which was determined by averaging the magnetic field dependence of the background on both sides of the resonance. In Fig.4 the photosignal of the peak is shown as a function of intensity at a. wavelength of $\lambda=70.5 \mu \mathrm{m}$ for different electric voltages up to $2 \mathrm{~V}$. The relative photosignal $\Delta V / V$, and thus the optically generated free hole density depends on the electric bias voltage in contrast to a linear photoconductor. With rising bias voltage, $\Delta V / V$ substantially increases. This behaviour must be attributed to excited state impact ionization. In Fig.5 a double - log plot of the relative photosignal $\Delta V / V$ as function of intensity is displayed for three different bias 

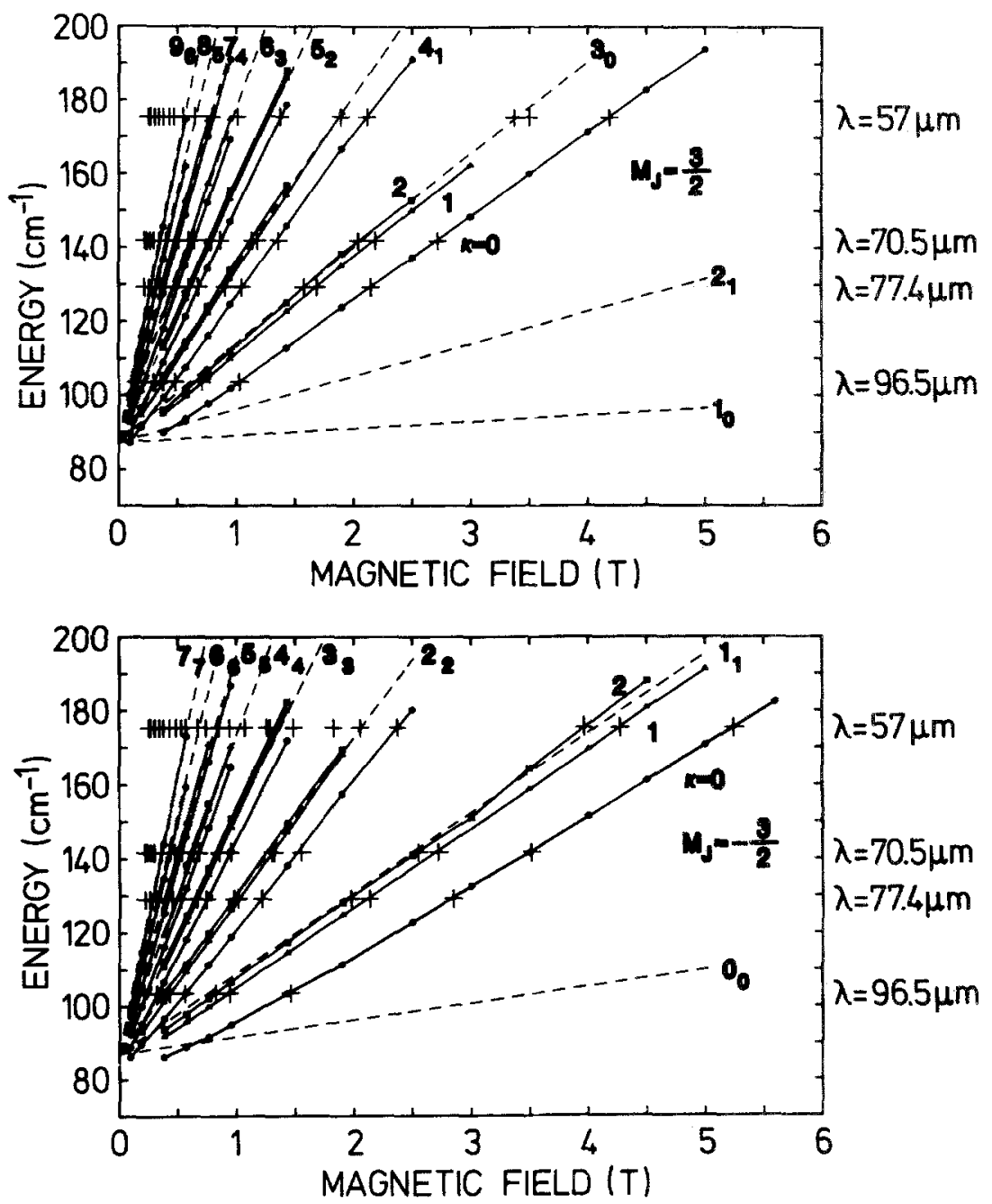

Fig.2 - Magnetic field dependence of all lines observed with Fourier spectroscopy (marked by dots) [7], and with laser magneto-spectroscopy of the present study (marked by crosses). Broken lines show the Landau levels with respect to the $B=0$ ionization energy of the boron acceptor.

voltages. This figure shows that the photosignal is never linear in the range of the applied intensities. The low intensity limit was determined by degrading signal to noise ratio. At high intensities 


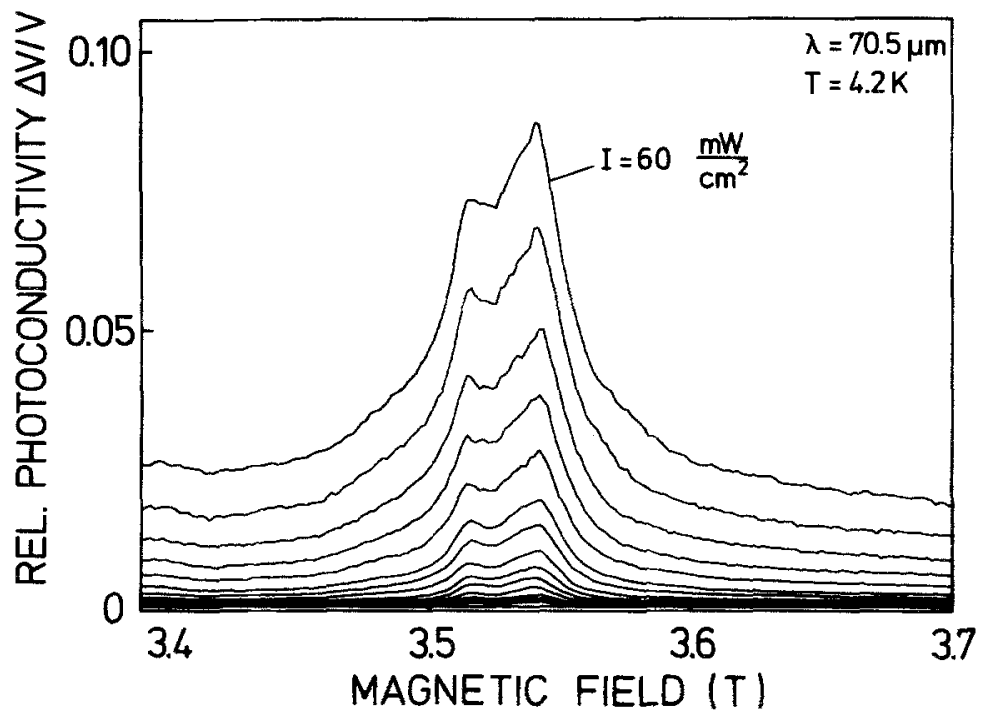

Fig.3 - Relative photoconductive signal $\Delta V / V$ of the transition to the quasi-bound Coulomb state $\left(1_{1}, 0\right)$ belonging to the light hole Landau level $1_{1}$ at an electric bias field of $2.86 \mathrm{~V} / \mathrm{cm}$. The fine structure of the resonance is attributed to energetically close lying final states of different $m$ but having the same $\kappa$.

the signal is proportional to the square root of the intensity (slope 1/2 in Fig.5), as already pointed out. Such an intensity dependence might be due to the saturation of an inhomogeneously broadened line or, like in the present case, group of lines, if the inhomogeneous width is larger than the unsaturated homogeneous linewidth [14].

If this situation applies we expect at low intensities a constant and intensity independent width representing the inhomogeneous linewidth. At high intensities the linewidth may eventually increase, if the power broadened homogeneous linewidth exceeds the inhomogeneous distribution of frequencies. However, as shown in Fig.6, the measurements yield a very different behaviour. The linewidth, determined from the high magnetic field wing of the spectral structure in Fig.3, rapidly increases at low intensities and shows a very small increase in the intensity range of square root dependence of the photoconductive signal. Thus the observed intensity dependence of the photoconductive signal cannot be due to the optical saturation of an inhomogeneously broadened line.

Another interesting point is the linewidth as function of the bias voltage shown in Fig.7. At constant intensity the linewidth 


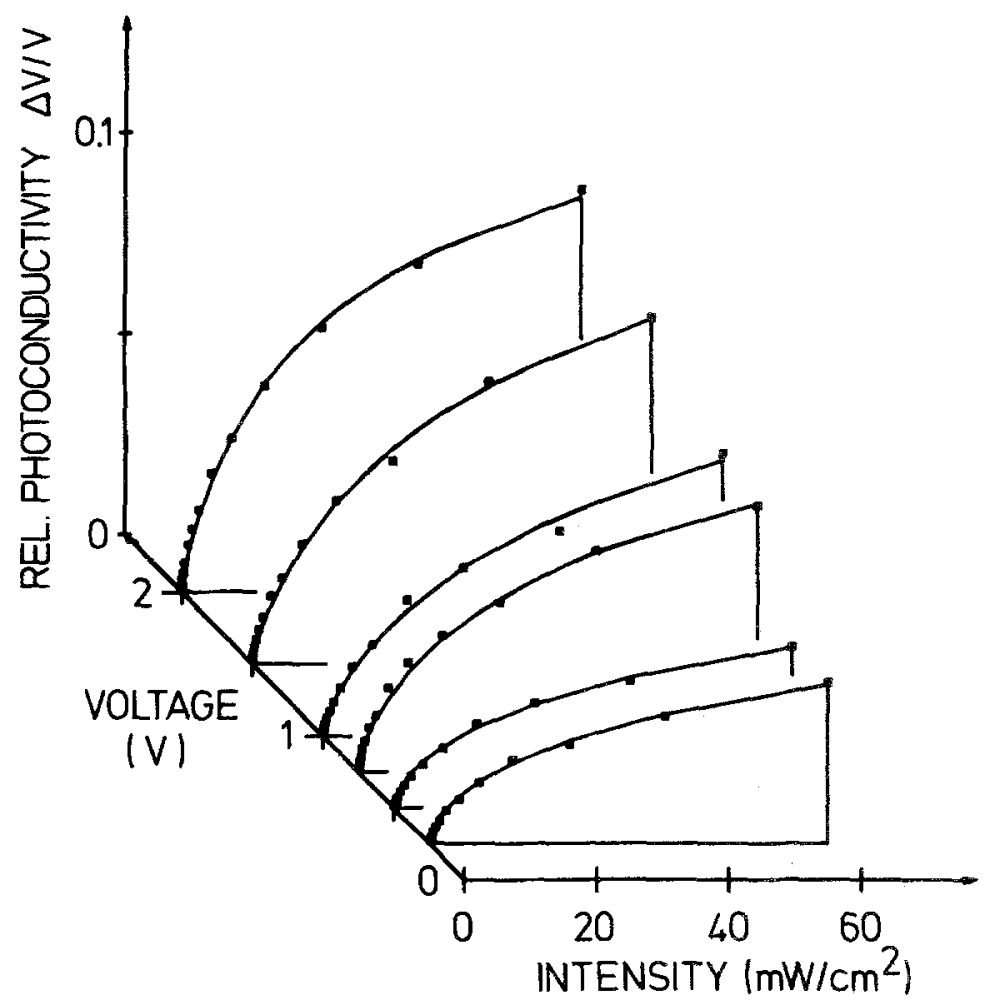

Fig.4 - Relative photoconductive signal $\Delta V / V$ as function of intensity for different electric bias fields. Squares are measured values, full lines are calculated curves.

significantly decreases with rising bias voltage. This effect is again an indication of excited state impact ionization. As the impact ionization is proportional to the number of free carriers, the peak signal is nonlinearly enhanced by photocarrier multiplication which in turn causes a narrowing of the line.

\section{Theory and Discussion}

The nonlinear characteristics of the photoconductive signal will be analyzed in terms of a rate equation model, taking into account the acceptor ground state, the valence band and inside the band the intermediate acceptor excited state i.e. the quasi-bound Coulomb 


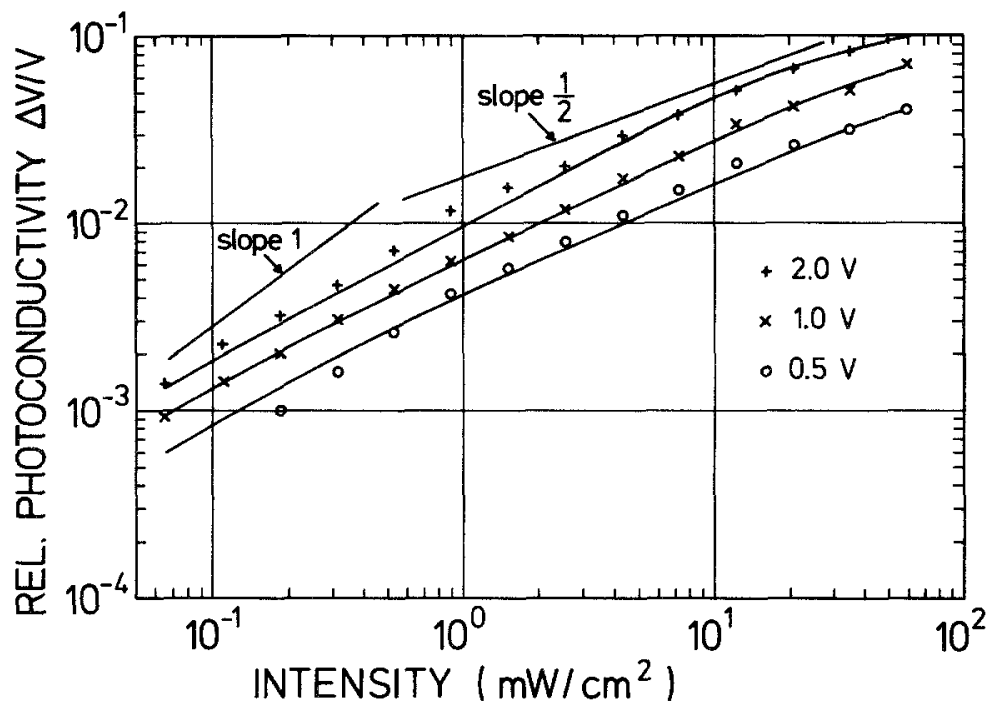

Fig.5 - Log-log plots of the photoconductive signal as function of the laser intensity for three different electric bias voltages. The square root dependence (slope one half) sets in at about $1 \mathrm{~mW} / \mathrm{cm}^{2}$ for the highest voltage. Full lines are calculated curves.

state belonging to the $1_{1}$ light hole Landau level at a magnetic field strength of $3.5 \mathrm{~T}$. The quasi-bound excited acceptor state lies below the $1_{1}$ Landau level, it is, however, located in the energy continuum of lower lying Landau subbands. Fig. 8 shows a schematic energy level diagramm corresponding to this situation; the hatched area indicates the continuum of free hole states. Generation and recombination processes being of importance at low temperatures are indicated by arrows and denoted by rate coefficients. Holes are optically excited from the acceptor ground state with a transition probability $\sigma F$, where $F$ is the photon flux density and $\sigma$ is the resonant absorption cross section. Thermal excitation of the upper acceptor state will be neglected because of the low temperature. Futhermore nonresonant direct photoionization of shallow acceptors will be also neglected, because in the range of applied intensities the resonant signal is substantially larger than the continuous photoconductivity background. Holes in the upper state are transferred to the valence band by thermal phonons or impact ionization. These processes are introduced by the kinetic coefficients $X_{2}^{S}$ and $X_{2}$. On the other hand holes may stick in the quasibound Coulomb state and relax to the ground state by nonradiative 


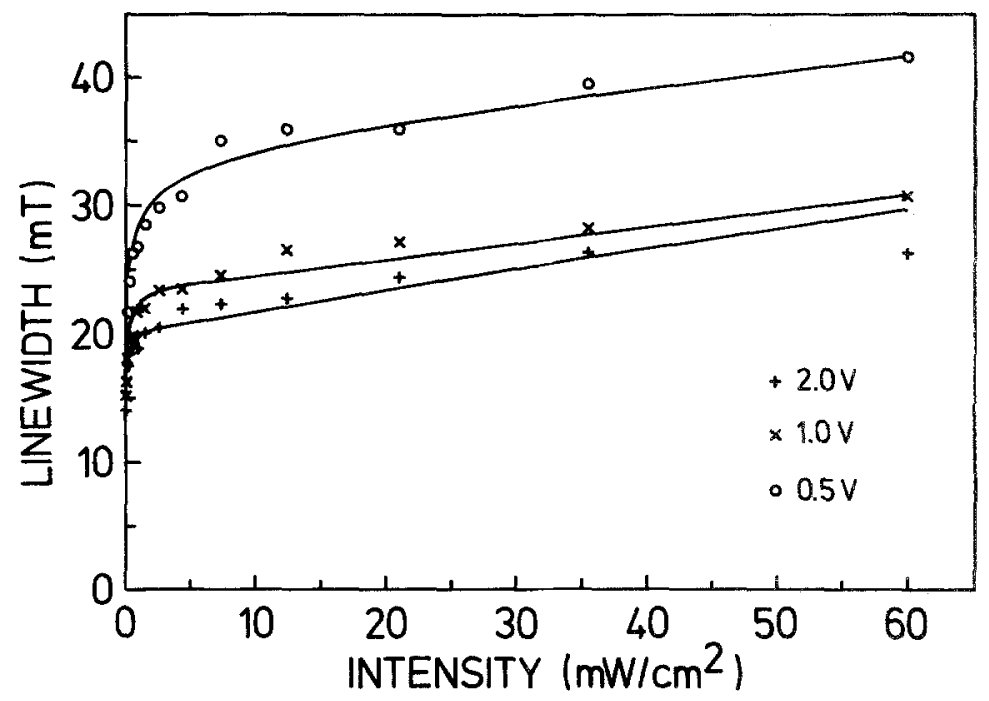

Fig.6 - Linewidth of the $\left(1_{1}, 0\right)$ transition as function of intensity for different bias voltages. Full lines are calculated curves.

transitions, $T_{2}^{S}=\tau_{2}^{-1}$, where $\tau_{2}$ is the corresponding transition time. Excited states of shallow impurities have typically rather long lifetimes [5], therefore additionally stimulated emission, $\sigma F$, must be considered connecting the upper acceptor level and the ground state. Impact ionization of the acceptor in the excited state must be taken into account in order to describe the observed voltage dependence of the relative photoconductivity. Free holes in different Landau levels may differently contribute to impact ionization. As details of this scattering process for valence band Landau levels are not known, we consider an average rate $X_{2} p p_{A}^{*}$, where $p$ is the total concentration of free holes and $p_{A}^{*}$ is the density of excited acceptors. Free holes are captured by ionized acceptors and recombine nonradiatively to the ground state. This process is taken into account by the kinetic coefficient $T_{1}^{S}$ leading to a recombination rate $T_{1}^{S} p\left(N_{D}+p\right)$. Stimulated emission by free holes may be neglected due to the large density of continuum states and due to the fact that free carriers generated at higher energies rapidly relax to the bottom of the band, thus the optical resonance condition is not met.

The rate equations in accordance to these considerations are 


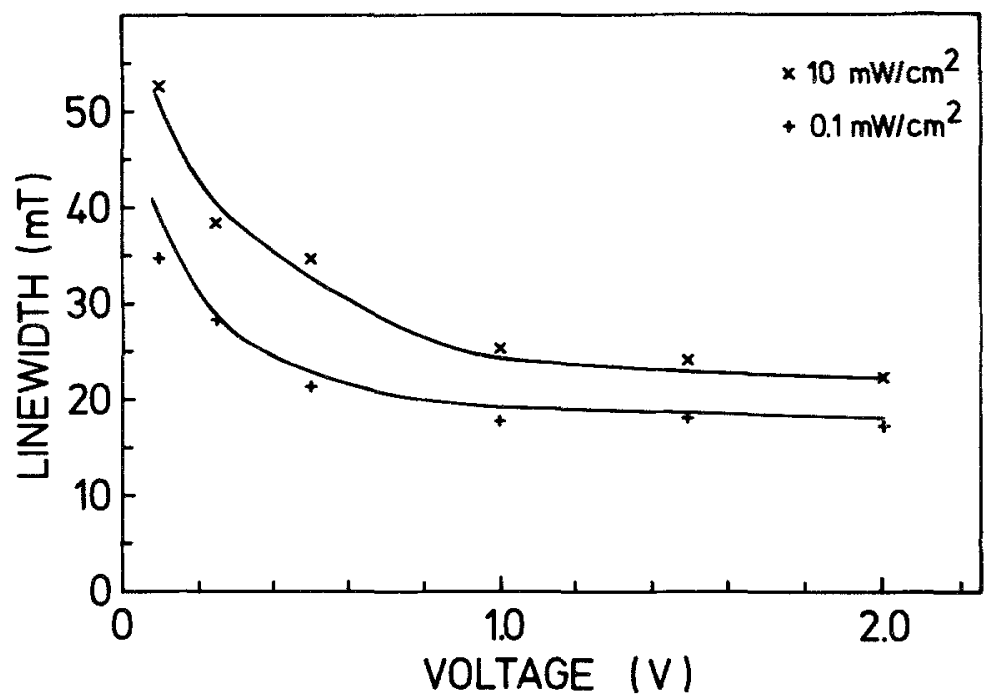

Fig.7 - Linewidth as function of the bias voltage for two different intensities. Full lines are calculated.

$$
\begin{gathered}
\frac{d p}{d t}=X_{2}^{S} p_{A}^{*}-T_{1}^{S} p n_{A}+X_{2} p_{A}^{*} p \\
\frac{d p_{A}^{*}}{d t}=\sigma F\left(p_{A}-p_{A}^{*}\right)-X_{2}^{S} p_{A}^{*}-T_{2}^{S} p_{A}^{*}-X_{2} p_{A}^{*} p
\end{gathered}
$$

where $p, p_{A}, p_{A}^{*}$ and $n_{A}$ are the densities of free holes, the $1 S_{3 / 2}$ acceptor ground state and the excited quasi-bound Coulomb state and the density of the ionized negativ acceptors, respectively. From the conservation of the total acceptor density $N_{A}^{*}=N_{A}-N_{D}=$ $p_{A}+p_{A}^{*}+p$, and the local neutrality condition $n_{A}=N_{D}+p$, one can simplify the rate equations. In steady state $\left(\frac{d}{d t}=0\right)$, this leads to a relation for the hole concentration by the balance of the carrier generation rate $g(p, F)$ and recombination rate $r(p)$

where

$$
g(p, F)=r(p)
$$

$$
g(p, F)=\left(N_{A}^{*}-p\right) \frac{i^{*} \sigma F+\xi \frac{p}{N_{A}^{*}} i^{*} \sigma F}{1+2 s^{*} \tau_{2} \sigma F+\xi i^{*} \frac{p}{N_{A}^{*}}}
$$

and

$$
r(p)=T_{1}^{S} p\left(N_{D}+p\right)
$$




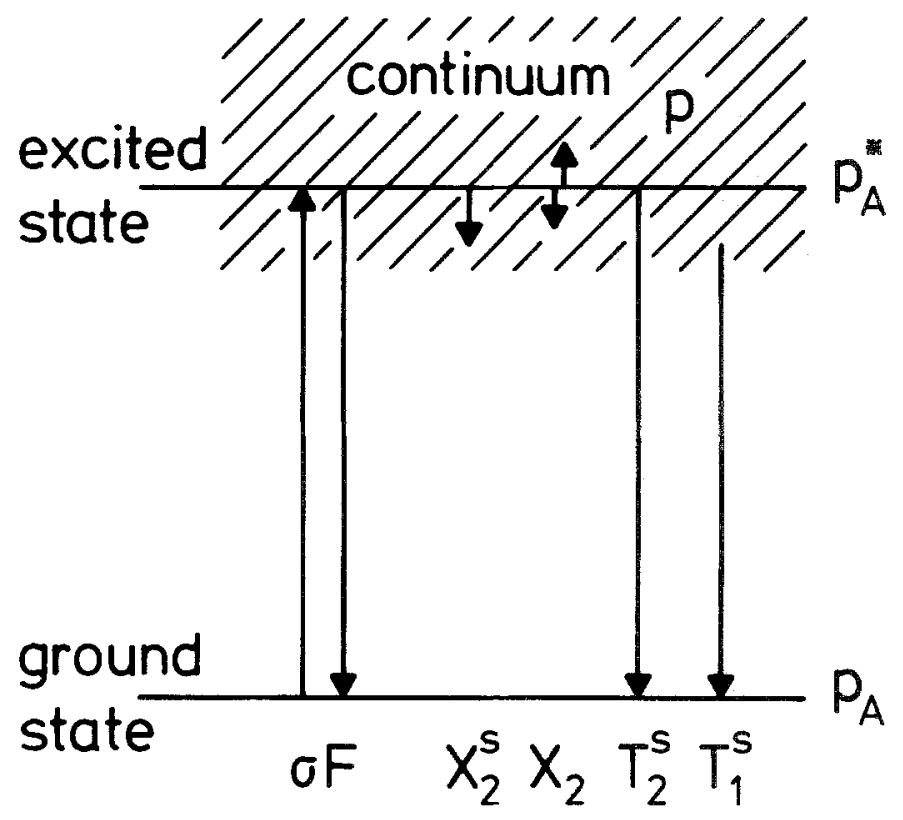

Fig.8 - Schematic energy level diagram and generation - recombination processes taken into account in the model described in the text.

In Eq.(4) $i^{*}$ and $s^{*}$ are the excited state thermal ionization probability $i^{*}=\frac{X_{2}^{S}}{X_{2}^{S}+T_{2}^{S}}$ and the corresponding sticking probability $s^{*}=1-p^{*}=\frac{T_{2}^{S}}{X_{2}^{S}+T_{2}^{S}}$, respectively. The parameter $\xi=\frac{X_{2} N_{A}^{*}}{X_{2}^{S}}$ is used to describe the impact ionization rate of excited states; $\xi$ represents the impact ionization probability per hole in the limit of fully ionized acceptors, $p=N_{A}^{*}$, in units of the thermal ionization propability $X_{2}^{S}$.

The intensity dependence of the carrier density $p$ is essentially controlled by the recombination rate $r(p)$. For materials with large compensation ratios, $\sigma F$ may approach $\tau_{2}^{-1}=T_{2}^{S}$ whereas $p$ remains still smaller than the concentration of compensating impurities, $p<<N_{D}$. In this case the recombination rate may be linearized, $r(p)=p T_{1}^{S} N_{D}$, leading to an exponential decay of free carriers with a recombination time $\tau_{1}=\left(T_{1}^{S} N_{D}\right)^{-1}$. Assuming neglectably small excited state impact ionization, $\xi<<1$, Eq.(3) gives the typical saturation behaviour 


$$
p=N_{A}^{*} \tau_{1} i^{*} \frac{\sigma F}{1+F / F_{S}}
$$

which is due to the depleting of the ground state by optical excitations. Here $F_{S}=\left(2 \sigma \tau_{e f f}\right)^{-1}$ is the saturation photon flux density with $\tau_{\text {eff }}=\frac{1}{2} i^{*} \tau_{1}+s^{*} \tau_{2}$ the effective relaxation time of optically excited carriers $[1,5]$. The free carrier concentration $p$, and thus the photoconductive signal $\Delta V / V$, depends linearly on $F$ for $F \ll F_{S}$ and assumes a constant, saturated value, $p_{\text {Sat }}=$ $N_{A}^{*} i^{*} \frac{\tau_{1}}{2 \tau_{e f f}}$ in the high intensity limit. This approximation has been used to evaluate resonant impurity photoconductivity in $\mathrm{n}$-GaAs at $4.2 K[3]$ and was also the basis of interpretation of high power absorption measurements $[1,2]$. It has been previously shown that at temperatures well below $4.2 \mathrm{~K}$ in $\mathrm{n}$-GaAs excited state impact ionization dominates the optically induced free carrier generation. This leads to an thresholdlike onset of the photoconductive signal, which has been discussed in detail in [7] and will not be worked out here.

This approach does not apply to the present case of low compensated samples. Over an intensity range of three orders of magnitude, the observed photosignal is neither linear for low intensities nor does it level at high intensities. Obviously linearization of the recombination rate is not justified. Due to the low density of compensating donors $N_{D}$, even if $\sigma F<<\tau_{2}^{-1}, p$ may exceed $N_{D}$, $p>N_{D}$, but still $p<<N_{A}^{*}$. In this limit and assuming small impact ionization $\xi<<1$, we obtain from Eq.(3) :

$$
p=\left(N_{A}^{*} N_{D} \tau_{1} i^{*} F\right)^{1 / 2}
$$

showing the square root dependence which was observed in a substantial range of intensities.

In order to take full account of the model defined in Eqs. (1) and (2), numerical solutions of Eq. (3) were determined and fitted to the experimental results by variing the parameters. The absorption cross section was determined by transmission spectroscopy yielding $\sigma=3.3 \cdot 10^{-14} \mathrm{~cm}^{2}$ at a magnetic field strength of $3.5 \mathrm{~T}$. The impact ionization coefficient $X_{2}$ as function of the bias voltage $V$ is described by the relation $X_{2}=X_{2}^{0} \exp \left(-\left(V_{0} / V\right)^{s}\right)$ derived from Shockley's "lucky electron" model [15]. The constant $V_{0}$ depends on the acceptor binding energy, the temperature, the mean free path of the holes and the geometry of the sample. The parameter $s$ may vary between one and two corresponding to the mean free path being independent of the electric field and proportional to the field, respectively [16]. Best fits were obtained using $X_{2}^{S}=1.63 \cdot 10^{4} \frac{1}{s}$, $T_{2}^{S}=2.52 \cdot 10^{6} \frac{1}{s}, T_{1}^{S}=2.8 \cdot 10^{-8} \frac{\mathrm{cm}^{3}}{\mathrm{~s}}, X_{2}^{0}=1.25 \cdot 10^{-7} \frac{\mathrm{cm}^{\frac{s}{3}}}{\mathrm{~s}}$, $V_{0}=1.1 \mathrm{~V}$ and $s=2$. The calculated results for the peak signal 
as function of intensity and bias voltage, the linewidth depending on intensity at constant voltage and the linewidth as function of the bias field are shown by the full lines in Fig. 4, 5, 6 and 7 in very good agreement to the measurements. The applied nonlinear generation-recomination-model nearly gives the excited state impact ionization narrowing of the linewidth. Thus photoconductivity measurements, which require a bias voltage across the sample, do not necessarily reproduce the intrinsic line shape of optical impurity transitions. Furthermore we point out that with the parameter $s=1$ no reasonable fit of the data could be obtained; $s=1$ has been frequently used to model the impact ionization probability $[4,7,8,17]$. From the kinetic coefficients we obtain the excited state thermal ionization probability $i^{*}=X_{2}^{S}\left(T_{2}^{S}+X_{2}^{S}\right)^{-1} \approx 6.4 \cdot 10^{-3}$, thus in the limit of vanishing impact ionization at zero or very low bias voltages not more than about $0.6 p c$ of the population of the metastable state is thermally transfered to the valence band. The majority of the holes stays at the acceptor and cascades across excited states within a mean time $\tau_{2}=\left(T_{2}^{S}\right)^{-1}=0.4 \mu$ s to the ground state by emitting acoustic phonons. This long transition time agrees well to time constants derived previously from the bandwidth of rf-modulated far-infrared photoconductivity in p-Ge [18]. In the framework of the present rate equation model, the excited acceptor state lifetime is given by $\tau^{*}=\left(X_{2}^{S}+T_{2}^{S}\right)^{-1}=\left(1-i^{*}\right) \tau_{2}$. Because of the very small ionization probability, $\tau^{*}$ is practically equal to the transition time $\tau_{2}$. We have, however, to keep in mind that a hole actually cascades across intermediate states to the ground state [19]. This cascade relaxation has been simplified by the onestep process described by $T_{2}^{S}$. The thermal transition rate from the optically populated excited state to intermediate states may be much faster than $T_{2}^{S} N_{A}^{*}$. Therefore $\tau^{*}$ gives only an upper limit of the lifetime which is expected to be significantly smaller. The real life time cannot be measured by optical saturation. Generally, saturation of an optical transition in a multilevel system yields the average transition time from the excited state to the initial state rather than the excited state life time. In many cases "life times" determined by saturation spectroscopy in extrinsic semiconductors (e.g. [1] and [2]) are artefacts of the underlying model and actually represent mean transition times ignoring the details of the transition kinetics.

\section{Conclusion}

In summary, we have investigated the nonlinear photoconductivity due to optical excitation to quasi-bound Coulomb states of light holes in the continuum of the light hole Landau levels. The optically generated free hole concentration exceeds the compensating shallow 
donor density at intensities above about 1 to $3 \mathrm{~mW} / \mathrm{cm}^{2}$, depending on the electrical bias field. This yields a free carrier recombination rate $r \sim p^{2}$, which causes a nonexponential decay of free hole concentration. In this case the resonant photoconductive signal is proportional to the square root of intensity and it is not possible to define a saturation intensity being independent of free hole density. The linewidth of the observed shallow acceptor transitions exhibit a pronounced narrowing upon increasing the electric bias field. This effect is attributed to impact ionization of optically excited states which substantially contributed to the resonant photoconduction signal at bias voltages well below the threshold of impact ionization breakdown. A nonlinear carrier generation- recombination model incorporating these features has been treated and a set of kinetic parameters were determined which give good agreement between calculated and experimental results.

\section{Acknowledgement}

We thank E. E. Haller for provision of the Germanium crystal. Financial support by the Deutsche Forschungsgemeinschaft is gratefully acknowledged.

\section{REFERENCES}

[1] C.R. Pidgeon, A. Vass, G.R. Allan, W. Prettl and L. Eaves, Phys. Rev. Lett. 50, 1309 (1983).

[2] G.R. Allan, A. Black, C.R.Pidgeon, E. Gornik, W. Seidenbusch and P. Colter, Phys. Rev. B 31, 3560 (1985).

[3] W. Prettl, A. Vass, G.R. Allan and C.R. Pidgeon, Int. J. Infrared and Millimeter Waves 4, 561 (1983).

[4] M. Weispfenning, I. Hoeser, W. Böhm, W. Prettl and E. Schöll, Phys. Rev. Lett. 55, 754 (1985).

[5] J. Kaminski, J. Spector, W. Prettl and M. Weispfenning, Appl. Phys. Lett. 52, 233 (1988).

[6] Sh.M. Kogan and T.M. Lifshits, phys. stat. sol. a 39, 11 (1977).

[ 7] M. Weispfenning, F. Zach and W. Prettl, Int. J. Infrared and Millimeter Waves 9, 1153 (1988).

[ 8] E.Schöll, W. Heisel and W. Prettl, Z. Phys. B 47, 285 (1982).

[9] V.F. Gantmakher, B.L. Gelmont, V.N. Zverev and A.I.L. Efros, Sov. Phys. JEPT 57, 656 (1983).

[10] G. Jungwirt, R. Kropf, U.Roessler and W.Prettl, Solid St. Commun. 72, 17 (1989). 
[11] K. Suzuki and J.C. Hensel, Phys. Rev. B 9, 4184 (1974).

[12] J.Simola and J. Virtamo, J. Phys. B 11, 3309 (1978).

[13] H.-R. Trebin, U. Rössler and R. Ranvaud, Phys. Rev. B 20, 686 (1979).

[14] R.H. Pantell and H.E. Puthoff, Fundamentals of Quantum Electronics, New York, Wiley, 1969.

[15] W. Schockley, Solid State Electron. 2, 35 (1961).

[16] G.A. Baraff Phys. Rev. 128, 2507 (1962).

[17] E. Schöll, Nonequilibrium Phase Transitions in Semiconductors, Berlin, 1987.

[18] E.M. Gershenzon, G.N. Gol'tsman, V.V. Multanovskii and N.G. Ptitsina, Sov. Phys. JEPT 50, 728 (1979).

[19] M. Lax, Phys. Rev. 119, 1502 (1960). 